\title{
Preface: Special topic on Precise Catalysis Science and Technology
}

\author{
Wenjie Shen \\ State Key Laboratory of Catalysis, Dalian Institute of Chemical Physics, Chinese Academy of Sciences, Dalian 116023, China
}

Received May 27, 2021; accepted May 31, 2021; published online June 8, 2021

Citation: Shen W. Preface: Special topic on Precise Catalysis Science and Technology. Sci China Chem, 2021, 64: 1063-1064, https://doi.org/10.1007/s11426021-1038-4

Nanoparticles, clusters and single-atoms constitute the major types of metal catalysts. The size variation of metal species, known as size effect in heterogeneous catalysts, not only changed the geometric and electronic structure of metal atoms, but also altered their anchoring strength with the immobilizing supports, showing distinctively varied activities. A gradual accumulation of large amounts of experimental data and theoretical calculations has unambitiously verified that the size of the metal species played a crucial role in determining the catalytic performance with respect to the geometric and electronic structures of active sites. However, it remains challenging to identify the intrinsic activity of the nanoparticles, clusters and single-atoms. The precise control of metal dispersion, coordination environment, and the metal-support electronic structure would provide quantitative description of the catalytic mechanisms of metal nanoparticles, clusters, and single-atoms. Among them, metal clusters, containing few to tens or hundreds of atoms, are characterized by the exact formula and crystallographically structures, enabling to tailor the active sites and correlate the structure-reactivity relationship at atomic accuracy. Significant progress has been made in this field thanks to the advanced synthesis technique in atomic control of the clusters and their surroundings. The observed unique catalytic properties were linked to the atomic configurations of the specific cluster, which governed the adsorption and activation of the reacting molecules.

In this special topic on precise catalysis, we collected three research articles, one feature article and one minireview article, covering pure clusters, clusters deposited inside zeolites and clusters supported by oxides, and their applications in electrocatalysis, photocatalysis and thermal catalysis. The research article by Kong et al. [1] mediated the electronic structure of $\mathrm{Cu}$ clusters via $\mathrm{P}$ doping, which stabilized surface $\mathrm{Cu}^{\delta+}$ species and regulated the adsorbed strength of the reaction intermediate $\left({ }^{*} \mathrm{CO}\right)$, and thereby enhanced the activity for $\mathrm{C}_{2+}$ production during $\mathrm{CO}_{2}$ electro-reduction. Zhai et al. [2] reported a general approach to selectively deposit $\mathrm{FeO}_{x}$ species onto the Lewis acid sites in microporous ZSM5 by atomic layer deposition, and interestingly found that the resulting $\mathrm{Fe}-\mathrm{O}-\mathrm{Si}$ species were highly selective for the oxidation of cyclohexane to cyclohexanone. The minireview by Sun et al. [3] summarized the catalytic performance of the gold clusters with well-defined atomic numbers and tunable geometries in electrocatalytic and photocatalytic reactions, pointing out the importance of the geometrical and electronic structures as well as the capped ligands. The article by Ning et al. [4] examined the geometrical structure and chemical state of copper clusters over ceria and found that the density of surface oxygen vacancies (or defect sites) of the oxidesupport largely affected the dispersion of $\mathrm{Cu}$ species. The feature article by Li et al. [5] developed an effective strategy to create oxygen vacancies using gas-phase atomic $\mathrm{H}$, and identified the reactivity of hydrogen species over oxide surfaces.

All these studies have demonstrated the possibility to precisely fabricate the active sites over solid catalysts and provided a deep understanding in the structure-performance relationship at the atomic level.

1 Kong X, Wang C, Zheng H, Geng Z, Bao J, Zeng J. Sci China Chem, 2021, 64: 1096-1102 
2 Zhai L, Zhang B, Liang H, Wu H, Yang X, Luo G, Zhao S, Qin Y. Sci China Chem, 2021, 64: 1088-1095

3 Sun Y, Cai X, Hu W, Liu X, Zhu Y. Sci China Chem, 2021, 64: 1065-
1075

4 Ning J, Zhou Y, Shen W. Sci China Chem, 2021, 64: 1103-1110

5 Li Z, Huang W. Sci China Chem, 2021, 64: 1076-1087

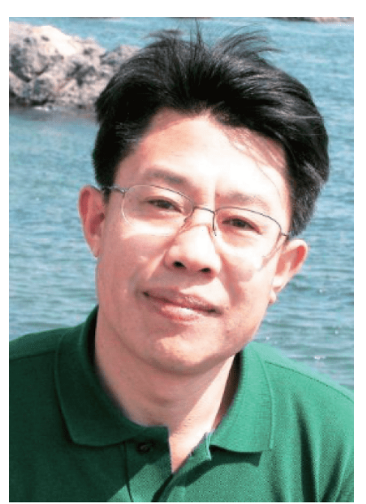

Wenjie Shen received his PhD degree from the Institute of Coal Chemistry, Chinese Academy of Sciences. He is currently a professor at the Dalian Institute of Chemical Physics, Chinese Academy of Sciences. His research interest includes synthesis of catalysts with controllable particle size and shape, analyses on the dynamic behavior of catalytic materials under or close to practical reaction conditions, and application of the nanostructured catalytic materials to chemical reactions that are potentially significant for energy and environmental processes. 